\title{
Right Utumi P.P.-Rings
}

\author{
ULRICH AlBRECHT
}

ABSTRACT - The class of right Utumi p.p.-rings plays a central role when developing a notion of torsion-freeness over non-commutative rings. This paper shows that it also arises naturally when considering divisible modules. Various notions of divisibility were introduced for modules over integral domains. We determine their relation in the non-commutative case and show that there are significant differences between this and the commutative setting. Finally, we determine large classes of semi-prime Goldie rings for which two or more of these notions coincide.

\section{Introduction.}

The straightforward attempt to extend the notion of torsion-freeness from integral domains to non-commutative rings encounters immediate difficulties. To overcome these, one can concentrate on either the computational or the homological properties of torsion-free modules. Goodearl and others took the first approach when they introduced the notion of a non-singular module [8]. A right $R$-module $M$ is non-singular if $Z(M)=0$ where $Z(M)=\{x \in M \mid x I=0$ for some essential right ideal $I$ of $R\}$ denotes the singular submodule of $M$. On the other hand, $M$ is singular if $Z(M)=M$. Moreover, a submodule $U$ of an $R$-module $M$ is $\mathcal{S}$-closed if $M / U$ is non-singular. Finally, $R$ is a right non-singular ring if $R_{R}$ is nonsingular. The right non-singular rings are precisely the rings which have a regular, right self-injective maximal right ring of quotients, which will be denoted by $Q^{r}$ (see [8] and [12] for details). Following [12, Chapter XI], $Q^{r}$ is a perfect left localization of $R$ if $Q^{r}$ is flat as a right $R$-module and the multiplication map $Q^{r} \otimes_{R} Q^{r} \rightarrow Q^{r}$ is an isomorphism. In particular, $Q^{r}$ is a perfect left localization of $R$ if and only if every finitely generated non-

(*) Indirizzo dell'A.: Department of Mathematics Auburn University, Auburn, AL 36849, U.S.A.

E-mail: albreuf@mail.auburn.edu 
singular right $R$-module can be embedded into a projective module ([8, Theorem 5.17] and [12, Corollary XII.7.3]). We call such a ring right strongly non-singular.

Hattori took the second approach by defining $M$ to be torsion-free if $\operatorname{Tor}_{1}^{R}(M, R / R r)=0$ for all $r \in R$ [9]. The classes of torsion-free and nonsingular right $R$-modules coincide if and only if $R$ is a right Utumi p.p.-ring without an infinite set of orthogonal idempotents [4, Theorem 3.7]. Here, $R$ is a right p.p.-ring if all principal right ideals of $R$ are projective. Moreover, a right non-singular ring $R$ is a right Utumi-ring if every $\mathcal{S}$-closed right ideal of $R$ is a right annihilator. The right and left Utumi-rings are precisely the right and left non-singular rings whose maximal right and left ring of quotients coincide (see [8, Theorem 2.38] and [12, Proposition XII.4.9]).

Section 2 investigates the structure of right Utumi p.p.-rings without an infinite set of orthogonal idempotents. Theorem 2.3 and Corollary 2.4 show that these rings can be viewed as lower triangular matrix rings. Closely related to the notion of torsion-freeness is that of divisibility where a right $R$-module $D$ is divisible if $\operatorname{Ext}_{R}^{1}(R / r R, D)=0$ for all $r \in R$. From [5, Section 2], one obtains that a right $R$-module $M$ is torsion-free if and only if its character module $M^{*}=\operatorname{Hom}_{\mathrm{Z}}(M, \mathrm{Q} / \mathrm{Z})$ is divisible. Moreover, if $R$ is a left p.p.-ring, then a left module $N$ is divisible if and only if $N^{*}$ is torsionfree. Therefore, it is not surprising that Utumi p.p.-rings also arise in the discussion of divisible modules (Theorem 3.2).

In Section 4, we look at other notions of divisibility. A right $R$-module $D$ is divisible in the classical sense if $D c=D$ for all regular elements $c$ of $R$. Finally, it is $h$-divisible if it is an epimorphic image of a direct sum of copies of $Q^{r}$. In case of domains, the notions of divisibility and divisibility in the classical sense coincide. Moreover, they also are equivalent to h-divisibility if $R$ is a countable integral domain ([7, Theorem VII.2.8] and [10]). Although we show in Sections 4 and 5 that this need not be the case if $R$ is non-commutative, the various notions of divisibility will, nevertheless, coincide if $R$ is a semi-prime right and left Goldie p.p.-ring whose classical ring of quotients is countably generated as a right and left $R$-module (Theorems 5.2 and 5.5).

\section{The Structure of Right Utumi P.P.-Rings.}

A ring $R$ is a Baer-ring if the right annihilator of a subset of $R$ is generated by an idempotent. In contrast to the p.p.-condition, being a 
Baer-ring is right-left symmetric. Our first result characterizes the right Utumi p.p.-rings without an infinite family of orthogonal idempotents in terms of their idempotents.

Proposition 2.1. A right non-singular ring $R$ is a right Utumi p.p.ring without an infinite set of orthogonal idempotents if and only if the following hold:

i) $R$ has finite right Goldie dimension.

ii) If $f \in Q^{r}$ is an idempotent, then there is an idempotent $e \in R$ with $e f=f$ and $f e=e$.

Proof. Suppose that $R$ is a right Utumi p.p.-ring without an infinite family of orthogonal idempotents. By [4], $R$ is a Baer-ring, and has finite right Goldie dimension. If $f \in Q^{r}$ is an idempotent, then $I=f Q^{r} \cap R$ is an $\mathcal{S}$-closed right ideal of $R$ since $R / I \cong\left[f Q^{r}+R\right] / f Q^{r} \subseteq Q^{r} / f Q^{r} \cong(1-f) Q^{r}$ is non-singular. Because $R$ is a right Utumi-ring, we obtain that $I$ is the right annihilator of a subset of $R$. Since $R$ is Baer, there is an idempotent $e \in R$ with $I=e R$. Then, $e Q^{r}=I Q^{r} \subseteq f Q^{r}$. Because $R$ is essential in $Q^{r}, I Q^{r}$ is an essential $R$-submodule of $f Q^{r}$. In view of the fact that $Q^{r}$ is semi-simple Artinian, $I Q^{r}$ is a direct summand of $f Q^{r}$, and hence $f Q^{r}=e Q^{r}$. But then $e f=f$ and $f e=e$.

Conversely, assume that the right non-singular $R$ satisfies the two conditions in the proposition. Clearly, $R$ contains no infinite set of orthogonal idempotents; and $Q^{r}$ is semi-simple Artinian by i) [12, Theorem XII.2.5]. It remains to show that $R$ is a right Utumi p.p.-ring. For this, it suffices to show that every $\mathcal{S}$-closed right ideal $I$ of $R$ is generated by an idempotent. Since $Q^{r}$ is semi-simple Artinian, there is an idempotent $f \in Q^{r}$ with $I Q^{r}=f Q^{r}$. Choose an idempotent $e \in R$ as in ii), and observe $e Q^{r}=f Q^{r}$. To show $I=e R$, observe that $e \in I Q^{r}$ yields that there exists an essential right ideal $J$ of $R$ with $e J \subseteq I$. Since $I$ is $\mathcal{S}$-closed in $R$, one has $e R \subseteq I$, and $I=e R \oplus[I \cap(1-e) R]$. However, $I \cap(1-e) R \subseteq e Q^{r} \cap(1-e) Q^{r}=0$.

A family $\left\{M_{i} \mid i \in I\right\}$ of right modules over a ring $R$ is semi-rigid if $\operatorname{Hom}_{R}\left(M_{i}, M_{j}\right) \neq 0$ implies $\operatorname{Hom}_{R}\left(M_{j}, M_{i}\right)=0$ for all $i \neq j$ in $I$. Modules $U$ and $V$ are quasi-isomorphic if there exist essential monomorphisms $U \rightarrow V$ and $V \rightarrow U$. Finally, if $S \subseteq R$, then $r_{R}(S)=\{r \in R \mid S r=0\}$ denotes the right annihilator of $S$ in $R$.

LEMMA 2.2. Let $R=S \times$ T be a right Utumi p.p.-ring without an infinite set of orthogonal idempotents. Then the same holds for the rings $S$ and $T$. 
Proof. Since $S$ is a subring of $R$, it does not contain an infinite set of orthogonal idempotents either. If $s \in S$, then $r_{R}\left(\left(s, 1_{T}\right)\right)=r_{S}(s) \times\{0\}$ is generated by an idempotent $f=(e, 0)$ of $R$ since $R$ is a right p.p.-ring. Clearly, $e$ is an idempotent of $S$ with $r_{S}(s)=e S$. Thus, $S$ is a right p.p.-ring.

Finally, if $I$ is an essential right ideal of $R$, then $I=I_{S} \times I_{T}$ where $I_{S}$ and $I_{T}$ are essential right ideals of $S$ and $T$ respectively. Therefore, whenever $J$ is a $\mathcal{S}$-closed right ideal of $S$, then $J \times T$ is a $\mathcal{S}$-closed right ideal of $R$. Since $R$ is a right Utumi-ring, there is a subset $X$ of $R$ with $J \times T=r_{R}(X)$. Choose a subset $Y$ of $S$ with $X=Y \times\{0\}$. Clearly, $J=r_{S}(Y)$, and $S$ is a right Utumi-ring.

THEOREM 2.3. Let $R$ be a right Utumi p.p.-ring without an infinite family of orthogonal idempotents. Then,

a) $R=R_{1} \times \ldots \times R_{m}$ where $R_{1} \ldots, R_{m}$ are indecomposable right Utumi-p.p.-rings such that, for all $i=1, \ldots, m, R_{i}=I_{i, 1} \oplus \ldots \oplus I_{i, n_{i}}$ for uniform right ideals $I_{i, 1}, \ldots, I_{i, n_{i}}$ of $R_{i}$.

b) $N(R)=0$ if and only if, for each $i=1, \ldots, m$, the right ideals $I_{i, j}$ and $I_{i, k}$ are quasi-isomorphic whenever $1 \leq j, k \leq n_{i}$.

Proof. Since $R$ is a right Utumi p.p.-ring without an infinite set of orthogonal idempotents, its maximal right ring of quotients, $Q^{r}$, is semisimple Artinian by Proposition 2.1. Moreover, $R$ is a Baer-ring by [4, Theorem 3.7]. Hence, [4, Lemma 3.5] yields that every $\mathcal{S}$-closed right ideal of $R$ is generated by an idempotent. Write $Q^{r}=Q_{1} \times \ldots \times Q_{m}$ where each $Q_{i}$ is a simple Artinian ring. Let $f_{1}, \ldots, f_{n}$ be the central idempotents of $Q^{r}$ associated with this decomposition, and consider the two-sided ideals $I_{i}=R \cap Q_{i}$ of $R$. Since $R / I_{i} \cong\left[R+Q_{i}\right] / Q_{i}$ is non-singular as a right $R$ module, $I_{i}$ is an $\mathcal{S}$-closed right ideal of $R$. Consequently, it is generated by an idempotent $e_{i} \in R$. Since $I_{i}$ is an essential submodule of $Q_{i}$, one has $f_{i} Q=Q_{i}=I_{i} Q=e_{i} Q$. Then, $f_{i}=e_{i} f_{i}=f_{i} e_{i}=e_{i}$ for $i=1, \ldots, n$. Therefore, $R=R_{1} \times \ldots \times R_{m}$ where $R_{i}=e_{i} R e_{i}$ is indecomposable as a ring since $e_{i} Q e_{i}$ is a simple ring. Moreover, $N(R)=N\left(R_{1}\right) \times \ldots \times N\left(R_{n}\right)$. By Lemma 2.2, each $R_{i}$ is a right Utumi p.p.-ring without an infinite set of orthogonal idempotents. Hence, we may assume for the remainder of the proof that $R$ is indecomposable as a ring.

Since $R$ has finite right Goldie dimension, there exists a non-zero uniform right ideal $U_{1}$ of $R$. If $V_{1}$ is the $\mathcal{S}$-closure of $U_{1}$ in $R$, then $V_{1}$ is a direct summand of $R$ because $R$ is a Baer and right Utumi-ring. Write $R=V_{1} \oplus W_{1}$. If $W_{1} \neq 0$, then $W_{1}$ contains an $\mathcal{S}$-closed non-zero uniform submodule $V_{2}$. Since $V_{2}$ is a direct summand of $R$, we obtain 
$R=V_{1} \oplus V_{2} \oplus W_{2}$ for some right ideal $W_{2}$ of $R$. Since $R$ has finite Goldiedimension, this process has to stop, and $R=V_{1} \oplus \ldots \oplus V_{\ell}$ for some $\ell<\omega$ and uniform right ideals $V_{1}, \ldots, V_{\ell}$. This proves a).

On $\left\{V_{1}, \ldots, V_{\ell}\right\}$, define $V_{i} \sim V_{j}$ if and only if $\operatorname{Hom}_{R}\left(V_{i}, V_{j}\right) \neq 0$ and $\operatorname{Hom}_{R}\left(V_{j}, V_{i}\right) \neq 0$. Since the $V_{i}$ 's are uniform, each $V_{i}$ is a non-singular right $R$-module of Goldie dimension 1 . Therefore, every non-zero submodule of $V_{i}$ is essential in $V_{i}$. Moreover, if $U$ is a $\mathcal{S}$-closed submodule of $V_{1}$, then $1=\operatorname{dim} V=\operatorname{dim} U+\operatorname{dim} V / U$ yields $U=0$ or $U=V$. Hence, every non-zero map $\alpha: V_{i} \rightarrow V_{j}$ is an essential monomorphism. Therefore, $V_{i} \sim V_{j}$ if and only if $V_{i}$ and $V_{j}$ are quasi-isomorphic. In particular, $\sim$ is an equivalence relation. Without loss of generality, one may assume that there is $m \leq \ell$ such that $V_{1}, \ldots, V_{m}$ are the distinct representatives of the equivalence classes of $\sim$. For $j=1, \ldots, m$, set $I_{j}=\oplus\left\{V_{i} \mid V_{i} \sim V_{j}\right\}$. Since non-zero maps between the $V_{k}$ 's are essential monomorphism, $R=I_{1} \oplus \ldots \oplus I_{m}$ such that $\left\{I_{1}, \ldots, I_{m}\right\}$ is a semi-rigid family such that, for each $j, I_{j}=I_{j, 1} \oplus \ldots \oplus I_{j, n_{j}}$ with each $I_{j, k}$ uniform and $I_{j, k}$ and $I_{j, \ell}$ are quasi-isomorphic for all $k$ and $\ell$. Select a family $\left\{e_{1}, \ldots, e_{n}\right\}$ of orthogonal idempotents of $R$ with $I_{i}=e_{i} R$.

Suppose that $R$ is semi-prime. If $\operatorname{Hom}_{R}\left(I_{j}, I_{k}\right) \neq 0$ for some $j \neq k$, then $\operatorname{Hom}_{R}\left(I_{k}, I_{j}\right)=0$ by the semi-rigidity condition. Because $\operatorname{Hom}_{R}\left(I_{j}, I_{k}\right) \neq 0$, there is $r \in R$ with $0 \neq r I_{j} \subseteq I_{k}$. In particular, $e_{k} r e_{j} \neq 0$. In view of the fact that $N(R)=0$, we have $e_{k} r e_{j} R e_{k} r e_{j} \neq 0$. If $s \in R$ satisfies $e_{j} s e_{k} \neq 0$, then left multiplication by $e_{j} s e_{k}$ induces a non-zero endomorphism $\alpha$ of $R$ with $\alpha\left(I_{k}\right) \subseteq I_{j}$, which contradicts $\operatorname{Hom}_{R}\left(I_{k}, I_{j}\right)=0$. Therefore, $\operatorname{Hom}_{R}\left(I_{j}, I_{k}\right)=0$ whenever $j \neq k$.

If there is $r \in R$ with $r I_{j} \not \subseteq I_{j}$ for some $j$, then we can find $k \neq j$ such that $e_{k} r x \neq 0$ for some $x \in I_{j}$. Thus, multiplication from the left by $e_{k} r$ induces a non-zero map $I_{j} \rightarrow I_{k}$, a contradiction. Therefore, each $I_{j}$ is a two-sided ideal of $R$. Since $R$ is indecomposable, we have $m=1$.

Conversely, suppose that $R$ has a decomposition of the stated form. Again, we may assume $m=1$. Write $R=J_{1} \oplus \ldots \oplus J_{\ell}$ where each $J_{i}$ is a uniform right ideal of $R$, and $J_{j}$ and $J_{k}$ are quasi-isomorphic for all $j$ and $k$. Select orthogonal idempotents $d_{1}, \ldots, d_{\ell}$ of $R$ such that $J_{j}=d_{j} R$. If $N(R) \neq 0$, then there exists a non-zero $a \in R$ such that $a R a=0$. Select $i \in\{1, \ldots, \ell\}$ with $d_{i} a \neq 0$. Moreover, there exists $k$ such that $d_{i} a d_{k} \neq 0$. Thus, left multiplication by $d_{i} a$ is a non-zero map from $J_{k}$ to $J_{i}$. Since $\operatorname{Hom}_{R}\left(J_{i}, J_{k}\right) \neq 0$, there is $s \in R$ such that $0 \neq s J_{i} \subseteq J_{k}$. Because non-zero maps between the $J_{j}$ 's are monomorphisms, left multiplication by $d_{i} a s$ is a one-to-one map from $J_{i}$ to $J_{i}$. Thus, $d_{i} a s d_{i} a \neq 0$, and the same holds for $\operatorname{asd}_{i} a$, a contradiction. 
COROLlaRY 2.4. Let $R$ be a right Utumi p.p.-ring without an infinite family of orthogonal idempotents. Then, $R \cong R_{1} \times \ldots \times R_{m}$ where each $R_{i}$ is an indecomposable ring which is isomorphic to a lower triangular matrix ring over rings $R_{i, 1}, \ldots, R_{i, n_{i}}$ such that each $R_{i, j}$ is a prime ring.

Proof. By Theorem 2.3, we may assume that $R$ is indecomposable. Observe that $Q^{r}(R) \cong \operatorname{Mat}_{\ell}(D)$ for some division algebra $D$. Using the notation of Theorem 2.3, write $R=I_{1} \oplus \ldots \oplus I_{m}$ such that $\left\{I_{1}, \ldots, I_{m}\right\}$ forms a semi-rigid family. We actually may assume that $I_{1}, \ldots, I_{m}$ are arranged in such a way that $\operatorname{Hom}_{R}\left(I_{j}, I_{k}\right)=0$ if $j<k$. Setting $M_{j k}=\operatorname{Hom}_{R}\left(I_{j}, I_{k}\right)$, one obtains that $R$ is isomorphic to the ring of matrices with entries from $M_{i j}$. Clearly, this is a lower triangular matrix ring. Furthermore, $R_{i}=\operatorname{Hom}_{R}\left(I_{i}, I_{i}\right)$ has a full matrix ring over $D$ as its maximal right ring of quotients.

CoRoLlary 2.5. Let $R$ be a right and left Utumi-p.p.-ring without an infinite family of orthogonal idempotents, and $A$ an essential submodule of $Q^{r}$ which is also a submodule of ${ }_{R} Q^{r}$. Then, $A=A_{1} \oplus \ldots A_{m}$ such that

a) $\left\{A_{1}, \ldots, A_{m}\right\}$ is semi-rigid.

b) For all $i=1, \ldots, m, A_{i}=A_{i, 1} \oplus \ldots \oplus A_{i, n_{i}}$ where each $A_{i, j}$ is uniform; and $A_{i, j}$ and $A_{i, k}$ are quasi-isomorphic for all $i=1, \ldots, m$ and all $1 \leq j, k \leq n_{i}$

Proof. Observe that $S=\operatorname{End}_{R}\left(A_{R}\right)$ is a subring $Q^{r}$ which contains $R$. By [1, Proposition 3.1] and Proposition 2.1, $S$ contains primitive idempotents $e_{i, j}$ such that $e_{i, j} S$ has the desired property. Let $A_{i, j}=e_{i, j} A$.

\section{Divisible Modules.}

Although the class of divisible modules is closed with respect to epimorphic images if $R$ is an integral domain, this does not hold in our more general setting:

LEMma 3.1. A ring $R$ is a right p.p.-ring if and only if the class of divisible right $R$-modules is closed with respect to epimorphic images. 
Proof. Let $R$ be a right p.p.-ring, and consider a submodule $U$ of a divisible module $D$. For all $r \in R$, we have an exact sequence

$$
0=\operatorname{Ext}_{R}^{1}(R / r R, D) \rightarrow \operatorname{Ext}_{R}^{1}(R / r R, D / U) \rightarrow \operatorname{Ext}_{R}^{2}(R / r R, U)=0
$$

from which we obtain that $D / U$ is divisible.

Conversely, suppose that epimorphic images of divisible modules are divisible. Then, $\operatorname{Ext}_{R}^{1}(R / r R, E / U)=0$ for all submodules $U$ of an injective module $E$ and all $r \in R$. We therefore have the exact sequence

$$
\operatorname{Hom}_{R}(R, E / U) \rightarrow \operatorname{Hom}_{R}(r R, E / U) \rightarrow \operatorname{Ext}_{R}^{1}(R / r R, E / U)=0 .
$$

By [11, Lemma 4.22], $r R$ is projective.

For integral domains, the notions of divisibility and divisibility in the classical sense obviously coincide. Although all divisible modules are divisible in the classical sense in the non-commutative setting, the converse may fail in view of the last result.

THEOREM 3.2. The following are equivalent for a right non-singular $\operatorname{ring} R$ :

a) $R$ is a right Utumi p.p.-ring without an infinite set of orthogonal idempotents.

b) $Q_{R}^{r}$ is torsion-free and $D \in_{R} \mathcal{M}$ is divisible if and only if $M \otimes_{R} D=0$ for all singular $M \in \mathcal{M}_{R}$.

c) ${ }_{R} Q^{r}$ is divisible, and $D \in \in_{R} \mathcal{M}$ is divisible if and only if $I D=D$ for all essential right ideals $I$ of $R$.

Proof. $a) \Rightarrow c$ ): If $D$ is a divisible left $R$-module, then $D^{*}=$ $=\operatorname{Hom}_{Z}(D, Q / Z)$ is a torsion-free right $R$-module in the sense of Hattori [5]. Since $R$ is a right Utumi p.p.-ring without an infinite set of orthogonal idempotents, $D^{*}$ is non-singular by [4]. If there is an essential right ideal $I$ of $R$ with $D / I D \neq 0$, then there exists a non-zero morphism $\phi: D / I D \rightarrow \mathrm{Q} / \mathrm{Z}$. We view $\phi$ as a non-zero map $D \rightarrow \mathrm{Q} / Z$ with $\phi(I D)=0$. Then, $[\phi r](d)=$ $=\phi(r d)=0$ for all $r \in I$, so that $\phi \in Z\left(D^{*}\right)=0$, a contradiction.

Conversely, suppose $D=I D$ for all essential right ideals $I$ of $R$. For $\alpha \in Z\left(D^{*}\right)$, there is an essential right ideal $J$ of $R$ such that $\alpha J=0$. But then $\alpha(J D)=0$, and $D^{*}$ is non-singular. By [4], this means that $D^{*}$ is torsion-free, from which we get that $D$ is divisible [5]. Finally, $R$ has finite right Goldiedimension. Therefore, $Q^{r}$ is semi-simple Artinian by [12, Theorem XII.2.5], and $I Q^{r}=Q^{r}$ for all essential right ideals $I$ of $R$ by [8, Proposition 2.32]. 
$c) \Rightarrow b$ ): Since the condition $D=I D$ is preserved by epimorphic images, the class of divisible left $R$-modules is closed with respect to epimorphic images. By Lemma 3.1, $R$ is a left p.p.-ring. As in [5], we obtain that ${ }_{R} D$ is divisible if and only if $D^{*}$ is torsion-free. Since $Q^{r}$ is divisible as a left $R$-module by c), we obtain that $\left(Q^{r}\right)^{*}$ is torsion-free. Moreover, $(I \cap R) Q^{r}=Q^{r}$ for all essential right ideals $I$ of $Q^{r}$. By [8, Proposition 2.32], $Q^{r}$ is semi-simple Artinian.

Let $S_{1}, \ldots, S_{n}$ be the pairwise non-isomorphic simple right $Q^{r}$-modules. Write $Q^{r}=R_{1} \times \ldots \times R_{n}$ where $R_{i}=\operatorname{Mat}_{n_{i}}\left(D_{i}\right)$ and $D_{i}$ is the division algebra $\operatorname{End}_{R}\left(S_{i}\right)$. Since $\left({ }_{R} Q^{r}\right)^{*}$ is a right $Q^{r}$-module, we obtain a decomposition $\left({ }_{R} Q^{r}\right)^{*}=U_{1} \oplus \ldots \oplus U_{n}$ where each $U_{i}$ is an $R_{i}$-module with $U_{j} R_{i}=0$ for $i \neq j$. Therefore, $U_{j} \cong \oplus_{m_{j}} S_{j}$ for some suitable $m_{j}<\omega$. Suppose that $m_{j}=0$ for some $j$. Let $\phi \in\left(Q^{r}\right)^{*}$, and consider $x \in Q^{r}$ and $q_{j} \in R_{j}$. Since $\left(Q^{r}\right)^{*} R_{j}=0$, we have $0=\left[\phi q_{j}\right](x)=\phi\left(q_{j} x\right)$. Thus, $\phi\left(R_{j}\right)=\phi\left(R_{j} Q^{r}\right)=0$. This is only possible if $R_{j}=0$, a contradiction. Hence, $m_{j}>0$ for all $j$. Since $\left({ }_{R} Q^{r}\right)^{*}$ is torsionfree, the same holds for $S_{1}, \ldots, S_{n}$, and, therefore, for $Q_{R}^{r}$ too.

Furthermore, if $D$ is a left $R$-module and $J$ is a right ideal of $R$, then the standard homological arguments show $(R / J) \otimes_{R} D \cong D / J D$. Therefore, $D$ is divisible if and only if $(R / I) \otimes_{R} D=0$ for all essential right ideals $I$ of $R$ because of c). Since every singular right $R$-module $M$ is the epimorphic image of $\oplus_{J} R / I_{j}$ for essential right ideals $I_{j}$ of $R$, we have $M \otimes_{R} D=0$ for every divisible left module $D$.

$b) \Rightarrow a)$ : By [4], it suffices to show that the classes of torsion-free and non-singular right $R$-modules coincide. Using $\mathrm{b}$ ), we obtain that epimorphic images of divisible left $R$-modules are divisible, and $R$ is a left p.p.ring. By [4], all non-singular right $R$-modules are torsion-free.

Conversely, consider a torsion-free right $R$-module $M$, and an essential right ideal $I$ of $R$. Since $M^{*}$ is a divisible left module [5], $I M^{*}=M^{*}$ because of $0=(R / I) \otimes_{R} M^{*} \cong M^{*} / I M^{*}$ by b). Suppose that there is $\phi \in M^{* *}$ with $\phi I=0$. For $x \in M^{*}$, there are $r_{1}, \ldots, r_{n} \in I$ and $y_{1}, \ldots, y_{n} \in M^{*}$ such that $x=r_{1} y_{1}+\ldots r_{n} y_{n}$. Then, $\phi(x)=\phi\left(r_{1} y_{1}\right)+\ldots+\phi\left(r_{n} y_{n}\right)=\left[\phi r_{1}\right]\left(y_{1}\right)+\ldots+$ $+\left[\phi r_{n}\right]\left(y_{n}\right)=0$. Therefore, $M^{* *}$ is non-singular, and the same holds for its submodule $M$. By [4], $R$ is a ring as in a).

CoRollary 3.3. Let $R$ be a right Ore-ring which is a right Utumi, right p.p-ring without an infinite set of orthogonal idempotents. Then, $Q_{c l}$ is a divisible left $R$-module if and only if $R$ is semi-prime.

Proof. If $Q_{c l}$ is divisible, then $I Q_{c l}=Q_{c l}$ for all essential right ideals $I$ of $R$ by Theorem 3.2. Thus, there are a regular element $c$ of $R$ and $x \in I$ with $x c^{-1}=1$, i.e. $c \in I$. By [8, Theorem 3.35], $R$ is semi-prime. 
A right $R$-module $D$ is absolutely pure if $\operatorname{Ext}_{R}^{1}(M, D)=0$ for all finitely presented modules $M$.

COROLLARY 3.4. The following are equivalent for a strongly nonsingular right Utumi-ring $R$ without an infinite set of orthogonal idempotents:

a) $R$ is right semi-hereditary.

b) All divisible right $R$-modules are absolutely pure.

Proof. $a) \Rightarrow b$ ): Let $D$ be a divisible right $R$-module, and $r \in R$. As in the proof of Theorem 3.2, we use [5] to obtain that $D^{*}$ is a torsion-free left $R$ module in the sense of Hattori. By [4], $D^{*}$ is flat since $R$ is right semihereditary. Then, $D^{* *}$ is injective, and $D$ is a pure submodule of an injective module. However, the class of absolutely pure modules is closed with respect to pure submodules.

$b) \Rightarrow a$ ) is established using arguments similar to the ones used in the proof of Lemma 3.1.

\section{The Singular Submodule of a Divisible Module.}

Over an integral domain $R$, the h-divisible modules are the epimorphic images of injective modules. This does not have to hold in our setting:

THEOREM 4.1. The following are equivalent for a right non-singular $\operatorname{ring} R$ :

a) $R$ has finite right Goldie-dimension.

b) A module is h-divisible if and only if it an epimorphic image of an injective module.

c) Non-singular h-divisible modules are injective.

Furthermore, $Z(D)$ is a direct summand of $D$ whenever $D$ is an h-divisible module over a finite dimensional right non-singular ring.

Proof. $a) \Rightarrow b$ ): Suppose that $U$ is a submodule of an injective module $E$, and consider an epimorphism $\pi: R^{(I)} \rightarrow E$ for some index-set $I$. It extends to an epimorphism $\bar{\pi}: Q^{r(I)} \rightarrow E$. Thus, $E / U$ is h-divisible. Conversely, observe that $Q^{r}$ is $\Sigma$-injective since $R$ has finite right Goldie-dimension. 
b) $\Rightarrow c$ ): Let $D$ be a non-singular h-divisible module. By b), there are an injective module $E$ and an epimorphism $\phi: E \rightarrow D$. As before, we can find an index-set $I$ and an epimorphism $\pi: Q^{r(I)} \rightarrow E$. We define a $Q^{r}$-module structure on $D$, which is compatible with its $R$-module structure, as follows: Whenever $q \in Q^{r}$ and $d \in D$, select $x \in Q^{r(I)}$ with $\phi \pi(x)=d$, and set $d * q=\phi \pi(x q)$. To see that this is well-defined, consider $y \in Q^{r(I)}$ with $\phi \pi(y)=d$. There is an essential right ideal $J$ of $R$ with $q J \subseteq R$. For all $r \in J$, we have $[\phi \pi(x q)-\phi \pi(y q)] r=[\phi \pi(x)-\phi \pi(y)] q r=0$. Since $D$ is non-singular, $\phi \pi(x q)=\phi \pi(y q)$. Finally, let $d^{\prime} \in D$ and $s \in R$. If $x^{\prime} \in Q^{r(I)}$ with $\phi \pi\left(x^{\prime}\right)=d^{\prime}$, then $d^{\prime} * s=\phi \pi\left(x^{\prime} s\right)=\phi \pi\left(x^{\prime}\right) s=d^{\prime} s$. In particular, $\phi \pi: Q^{r(I)} \rightarrow D$ becomes a $Q^{r}$-epimorphism. Suppose that we have already shown that $Q^{r}$ is a semisimple Artinian ring. Then, $D$ is a projective $Q^{r}$-module, and the epimorphism $\phi \pi$ splits. Consequently, $D$ is injective since it is isomorphic to a direct summand of the injective module $E$.

To see that $Q^{r}$ is semi-simple Artinian, it suffices to show that direct sums of copies of $Q^{r}$ are injective as right $R$-modules [12, Proposition XIII.3.3] since $Q_{R}^{r}$ is the injective hull of $R_{R}$ by [8, Chapter 2C]. To see this, we consider an index-set $J$, and apply the construction of the last paragraph to the h-divisible module $D=\oplus_{J} Q^{r}$. Observe that the two $Q^{r}$ module structures on $D$ actually coincide: Select $d \in D$ and $x \in Q^{r(I)}$ with $\phi \pi(x)=d$ as before. For $q \in Q^{r}$ select an essential right ideal $J$ with $q J \subseteq R$. For all $s \in J$, we have

$$
(d * q) s=[\phi \pi(x q)] s=\phi \pi(x(q s))=[\phi \pi(x)](q s)=d(q s)=(d q) s .
$$

Hence, $d * q=d q$ as desired. In particular, $(D, *)$ is a free $Q^{r}$-module, and the $Q^{r}$-epimorphism $\phi \pi: Q^{r(I)} \rightarrow D$ has to split, say $\phi \pi \alpha=1_{D}$ for some morphism $\alpha: D \rightarrow Q^{r(I)}$. But then, $\phi: E \rightarrow D$ splits, and $D \cong \oplus_{J} Q^{r}$ is injective for all index-sets $J$.

$c) \Rightarrow a$ ) is a direct consequence of the previously mentioned result of [12].

Finally, let $\lambda: D \rightarrow D / Z(D)$ be the canonical projection. If $\phi$ and $\pi$ are chosen as in the proof of $b) \Rightarrow c$ ), then $\lambda \phi \pi: Q^{r(I)} \rightarrow D / Z(D)$ splits, say $\lambda \phi \pi \psi=1_{D / Z(D)}$ for some $\psi: D / Z(D) \rightarrow Q^{r(I)}$. Hence, $Z(D)$ is a direct summand of $D$.

Although h-divisible modules are divisible in the classical sense, they need not be divisible:

COROLLARY 4.2. The following are equivalent for a right non-singular ring $R$ of finite right Goldie-dimension: 
a) $R$ is a right p.p.-ring.

b) Every h-divisible right $R$-module is divisible.

Proof. $\quad a) \Rightarrow b$ ): By Theorem 4.1, every h-divisible module $M$ is an epimorphic image of an injective module, and hence divisible by Lemma 3.1.

$b) \Rightarrow a$ ): Since every image of an injective is h-divisible by Theorem 4.1, we obtain that epimorphic images of injective modules are divisible. As in Lemma $3.1, R$ is a right p.p.-ring.

We say that a right $R$-module $M$ is weakly cotorsion if $\operatorname{Ext}_{R}^{1}\left(Q^{r}, M\right)=0$.

THEOREM 4.3. Let $R$ be a right Utumi p.p.-ring without an infinite set of orthogonal idempotents:

a) A right $R$-module $D$ is divisible if and only if $Z(D)$ is divisible and $D / Z(D)$ is injective.

b) $Z(D)$ is direct summand whenever $D$ is divisible if and only if all divisible $R$-modules are weakly cotorsion.

Proof. a) Let $r \in R$, and let $J$ be the $\mathcal{S}$-closure of $r R$ in $R$. Since $R$ is a right Utumi p.p.-ring, $J$ is a direct summand of $R$ by [4], say $R=P \oplus J$. Thus, $\operatorname{Ext}_{R}^{1}(R / r R,-) \cong \operatorname{Ext}_{R}^{1}(J / r R,-)$. In particular, the divisibility of $D$ guarantees $\operatorname{Ext}_{R}^{1}(J / r R, D)=0$. On the other hand, we have the exact sequence

$$
0=\operatorname{Hom}_{R}(J / r R, D / Z(D)) \rightarrow \operatorname{Ext}_{R}^{1}(J / r R, Z(D)) \rightarrow \operatorname{Ext}_{R}^{1}(J / r R, D)=0,
$$

from which we obtain $\operatorname{Ext}_{R}^{1}(R / r R, Z(D))=0$. Thus, $Z(D)$ is divisible.

To show that $D / Z(D)$ is injective, observe that it is divisible since $R$ is a right p.p.-ring. Therefore, we may assume that $D$ is non-singular, and the same holds for its injective hull $E$. Since $D$ is divisible, it is an RD-submodule of $E$. By [4], RD-submodules of non-singular modules are $\mathcal{S}$-closed. Thus, $E / D$ is non-singular, which contradicts the fact that $D$ is essential in $E$.

The converse is obvious since the class of divisible modules is closed with respect to extensions.

b) Assume that $Z(D)$ is a direct summand whenever $D$ is divisible, and consider an exact sequence $0 \rightarrow D \rightarrow M \rightarrow Q^{r} \rightarrow 0$. Then, $M$ is divisible, and $Z(M)=Z(D)$. By i), $M=Z(D) \oplus U$. Then, $D=Z(D) \oplus(U \cap D)$. Because of a), $U \cap D$ is injective. Hence, $U=(U \cap D) \oplus D_{1}$. Then, $M=Z(D) \oplus U=Z(D) \oplus(U \cap D) \oplus D_{1}=D \oplus D_{1}$ as desired. 
Conversely, suppose that divisible modules are weakly cotorsion. If $D$ is divisible, then $Z(D)$ is divisible, and $D / Z(D)$ is an injective module by a). Therefore, $\operatorname{Ext}_{R}^{1}\left(Q^{r}, Z(D)\right)=0$. Since $R$ is a finite dimensional ring, $D / Z(D)$ is a direct summand of $\oplus_{I} Q^{r}$ for some index-set $I$. However, $\operatorname{Ext}_{R}^{1}\left(\oplus_{I} Q^{r}, Z(D)\right)=0$, from which we obtain $\operatorname{Ext}_{R}^{1}(D / Z(D), Z(D))=0$.

We now give an example of a ring such that modules which are divisible in the classical sense need not be weakly cotorsion:

EXAMPLE 4.4. Let $F$ be a field, and $I$ an infinite index set. In $Q=F^{I}$ consider $R=F^{(I)}+F 1_{Q}$. By [8, Example 3.11], $R$ is a right non-singular ring whose maximal ring of quotients is $Q$. Since all regular elements of $R$ are units, every $R$-module is divisible in the classical sense. However, $Q$ is not projective, for otherwise it would be finitely generated by Sandomirski's Theorem [6, Proposition 8.24]. In this case, $\operatorname{dim}_{F} Q \leq\left(\operatorname{dim}_{F} R\right) \aleph_{0}<2^{|I|} \leq F^{|I|}=\operatorname{dim}_{F} Q$, a contradiction. Hence, we can find an $R$-module with $\operatorname{Ext}_{R}^{1}(Q, M) \neq 0$.

We conclude this section by looking at the semi-prime case:

COROLLARY 4.5. Let $R$ be a semi-prime right Goldie-ring. The following are equivalent for a non-singular module D:

a) $D$ is injective.

b) $D$ is divisible.

c) $D$ is divisible in the classical sense.

Proof. It remains to show $c) \Rightarrow a$ ): Let $J$ be an essential right ideal of $R$. Choose a regular element $c \in J$. For a map $\phi: J \rightarrow D$, consider $d=\phi(c)$ and select $x \in D$ with $x c=d$. If $\psi: R \rightarrow D$ is defined by $\psi(1)=x$, then $\left.\psi\right|_{c R}-\left.\phi\right|_{c R}=0$. Since $D$ is non-singular, and $J / c R$ is singular, $\left.\psi\right|_{J}=\phi$.

Furthermore, we obtain

Corollary 4.6. Let $R$ be a semi-prime right and left Goldie-ring.

a) $R$ is a p.p.-ring if and only if the classes of divisible modules coincides with the class of modules which are divisible in the classical sense.

b) All $R$-modules which are divisible in the classical sense are weakly cotorsion if and only if $Z(D)$ is a direct summand of $D$ whenever $D$ is divisible in the classical sense. 
Proof. a) In case that $R$ is a p.p.-ring, consider a right $R$-module $D$ which is divisible in the classical sense. By [5], it is enough to show that $D^{*}=\operatorname{Hom}_{Z}(D, Q / Z)$ is a non-singular left $R$-module. Suppose that $\phi: D \rightarrow Q / Z$ such that $c \phi=0$ for some regular element $c$ of $R$. For $d \in D$ select $d^{\prime} \in D$ such that $d=d^{\prime} c$. Then, $\phi(d)=\phi\left(d^{\prime} c\right)=c \phi\left(d^{\prime}\right)=0$.

Conversely, observe that the class of modules which are divisible in the classical sense is closed with respect to epimorphic images. By Lemma 3.1, $R$ is a right p.p.-ring.

b) Suppose that $D$ is divisible in the classical sense. Then the same holds for $D / Z(D)$, which is injective by Corollary 4.5. To see that $Z(D)$ is divisible in the classical sense, let $x \in Z(D)$. If $c \in R$ is regular, then there is $y \in D$ with $x=y c$. Since $x \in Z(D)$, there is a regular $d \in R$ with $x d=0$. Then, $y c d=0$ yields $y \in Z(D)$. We now can adapt the proof of Theorem 4.3 to establish b).

Example 5.6 will show that the condition that $R$ is semi-prime cannot be removed from the last result. The ring $R$ in Example 5.6 is divisible in the classical sense when viewed as a right module. However, it is not divisible. Otherwise, it would be absolutely pure by Corollary 3.4 since $R$ is hereditary. Because $R$ is right and left Noetherian, absolutely pure modules are injective, a contradiction.

\section{The Projective Dimension of $Q^{r}$.}

Our first result relates the splitting of $Z(D)$ to the projective dimension of $Q_{R}^{r}$ :

Proposition 5.1. Let $R$ be a right non-singular ring. If $Z(D)$ is a direct summand of every module $D$ which is divisible in the classical sense, then p.d. $\left(Q_{R}^{r}\right) \leq 1$.

Proof. Let $M$ be a right $R$-module with injective hull $E$. The singular module $E / M$ is divisible in the classical sense. Consider an exact sequence $0 \rightarrow E / M \rightarrow X \rightarrow Q^{r} \rightarrow 0$ of right $R$-modules. Since $E / M$ and $Q^{r}$ are divisible in the classical sense, the same holds for $X$. By hypothesis, the sequence splits, and $\operatorname{Ext}_{R}^{1}\left(Q^{r}, E / M\right)=0$. We obtain

$$
0=\operatorname{Ext}_{R}^{1}\left(Q^{r}, E / M\right) \rightarrow \operatorname{Ext}_{R}^{2}\left(Q^{r}, M\right) \rightarrow \operatorname{Ext}_{R}^{2}\left(Q^{r}, E\right)=0,
$$

and $Q^{r}$ has projective dimension at most 1 . 
We now give a non-commutative version of [7, Theorem VII.2.8]:

THEOREM 5.2. Let $R$ be a semi-prime, right and left Goldie-ring such that $(Q / R)_{R}$ is a direct sum of countably generated modules.

a) p.d. $\left(Q_{R}\right) \leq 1$.

b) A right $R$-module is h-divisible if and only if it is divisible in the classical sense and weakly cotorsion.

Proof. Let $U$ be a countably generated submodule of $Q_{R}$ containing $R$, say it is generated by $\left\{u_{n} \mid n<\omega\right\}$. Without loss of generality, we may assume $u_{0}=1$. We construct an ascending chain $\left\{V_{n}\right\}_{n<\omega}$ such that $V_{0}=R, V_{n}=d_{n}^{-1} R$ for some regular element $d_{n}$ of $R$ subject to the conditions $d_{0}=1$ and $d_{n+1}=c_{n} d_{n}$ and $u_{0}, \ldots, u_{n} \in V_{n}$. Since $R$ is a semi-prime right and left Goldie-ring, there is a regular element $g_{n} \in R$ such that $u_{n+1} \in g_{n}^{-1} R$. Select $c_{n}, r_{n} \in R$ with $c_{n}$ regular such that $d_{n} g_{n}^{-1}=c_{n}^{-1} r_{n}$ and set $d_{n+1}=c_{n} d_{n}=r_{n} g_{n}$. Then, $U \subseteq V=\cup_{n<\omega} V_{n}$. Since each $V_{n}$ is free, we have $p . d .(V) \leq 1$, and hence $p . d .(V / R) \leq 1$.

Now write $Q / R=\oplus_{I} A_{i} / R$ where each $A_{i}$ is a countably generated submodule of $Q_{R}$ containing $R$. Select $V_{i} \supseteq A_{i}$ as was constructed in the last paragraph. Then, $A_{i} / R$ is a direct summand of $V_{i} / R$, and hence has projective dimension at most 1 , and the same holds for $Q_{R}$.

Let $D$ be h-divisible, and select an epimorphism $\pi: Q^{(I)} \rightarrow D$ for some index-set $I$. Since $R$ has finite right Goldie-dimension, $Q^{(I)}$ is injective. Hence, we obtain a sequence

$$
0=\operatorname{Ext}_{R}^{1}\left(Q, Q^{(I)}\right) \rightarrow \operatorname{Ext}_{R}^{1}(Q, D) \rightarrow \operatorname{Ext}_{R}^{2}(Q, \text { ker } \pi)=0
$$

which is exact since p.d. $\left(Q_{R}\right) \leq 1$. Consequently, $D$ is weakly cotorsion.

Conversely, suppose that $D$ is weakly cotorsion and divisible in the classical sense. Consider the sequence

$$
\operatorname{Hom}_{R}(Q, D) \rightarrow \operatorname{Hom}_{R}(R, D) \rightarrow \operatorname{Ext}_{R}^{1}(Q / R, D) \rightarrow \operatorname{Ext}_{R}^{1}(Q, D)=0 .
$$

Once we have shown that $\operatorname{Ext}_{R}^{1}(Q / R, D)=0$, then $D$ is injective with respect to $0 \rightarrow R \rightarrow Q$. Consequently, $D$ is an image of a direct sum of copies of $Q$.

Now $\operatorname{Ext}_{R}^{1}(Q / R, D)=\Pi_{I} \operatorname{Ext}_{R}^{1}\left(A_{i} / R, D\right)$. Thus, it suffices to show that $\operatorname{Ext}_{R}^{1}\left(A_{i} / R, D\right)=0$. However, $Q / A_{i} \cong(Q / R) /\left(A_{i} / R\right) \cong \oplus_{j \neq i}\left(A_{j} / R\right)$ has projective dimension at most 1 . The exact sequence

$$
0=\operatorname{Ext}_{R}^{1}(Q, D) \rightarrow \operatorname{Ext}_{R}^{1}\left(A_{i}, D\right) \rightarrow \operatorname{Ext}_{R}^{2}\left(Q / A_{i}, D\right)=0
$$


yields $\operatorname{Ext}_{R}^{1}\left(A_{i}, D\right)=0$. Thus, $\operatorname{Ext}_{R}^{1}\left(A_{i} / R, D\right)=0$ provided that $D$ is injective with respect to $0 \rightarrow R \rightarrow A_{i}$. Since $A_{i} / R$ is countably generated, there are regular elements $u_{0}=1, u_{1}, \ldots$ of $R$ such that $W_{i}=\left\langle u_{n}^{-1} R \mid n<\omega\right\rangle$ contains $A_{i}$. If $\phi: R \rightarrow D$, then it is enough to show that $\phi$ can be extended to $W_{i}$. As before, there are regular elements $d_{0}, d_{1}, \ldots$ of $R$ such that $d_{n+1}=c_{n} d_{n}$ and $u_{n}^{-1} \in V_{n}=d_{n}^{-1} R$.

Let $\phi_{0}(1)=\phi(1)$, and assume that we have already defined maps $\phi_{i}: V_{i} \rightarrow D$ for $i=0, \ldots, n$ such that $\left.\phi_{i+1}\right|_{V_{i}}=\phi_{i}$ for $i=0, \ldots, n-1$. Now, $V_{n+1} / V_{n}=d_{n}^{-1} c_{n}^{-1} R / d_{n}^{-1} R \cong R / c_{n} R$. Since $D$ is divisible in the classical sense, $\operatorname{Ext}_{R}^{1}\left(R / c_{n} R, D\right)=0$, and hence $\phi_{n}$ extends to $\phi_{n+1}$ as desired. Therefore, $\phi$ can be extended to the submodule $\cup_{n<\omega} V_{n}$ of $Q_{R}$ which contains $W_{i}$.

However, decompositions of $Q^{r} / R$ need not be as nice as in the case that $R$ is an integral domain. For instance, it was shown in [7], that $Q / R=A / R \oplus B / R$ yields that $A$ and $B$ are subrings of $Q$. This need not hold in the non-commutative setting:

Proposition 5.3. Let $R$ be a right and left Utumi-ring of finite Goldiedimension whose maximal right and left ring of quotients is $Q$. If $Q / R=A / R \oplus B / R$ for submodules $A$ and $B$ of $Q_{R}$ containing $R$, then the following are equivalent:

a) $A$ and $B$ are subrings of $Q$.

b) $A$ and $B$ are submodules of ${ }_{R} Q$.

Proof. $b) \Rightarrow a$ ): Let $J$ be an essential right ideal of $R$. Since $R$ has finite Goldie dimension, $Q$ is a semi-simple Artinian ring, and $J Q=Q$ by [12, Proposition Theorem XII.2.5] and [8, Theorem 3.35]. For every $q \in Q$, we can find $r_{1}, \ldots, r_{n} \in J$ and $q_{1}, \ldots, q_{n} \in Q$ with $q=\sum_{i=1}^{n} r_{i} q_{i}$. For each $i$, there are $a_{i} \in A$ and $b_{i} \in B$ with $q_{i}=a_{i}+b_{i}$. Then, $q=\sum_{i=1}^{n} r_{i} a_{i}+\sum_{i=1}^{n} r_{i} b_{i} \in J A+J B$. Thus, $Q=J A+J B$. Moreover, if $q$ has been chosen from $A$, then $q-\left(\sum_{i=1}^{n} r_{i} a_{i}\right)=\sum_{i=1}^{n} r_{i} b_{i} \in A \cap B=R$ by b). Hence, $A=J A+R$.

Select $a \in A$, and choose an essential right ideal $I$ of $R$ with $a I \subseteq R$. Then, $a A=a(I A+R)=(a I)+a R \subseteq A$ by what has been shown in the last paragraph and $b$ ).

$a) \Rightarrow b$ ) is obvious.

EXAMPLE 5.4. Let $S$ be an integral domain with field of quotients $F$ which is not a field. The ring $R=M_{2} t_{2}(S)$ is a prime right and left Goldie- 
ring whose classical right and left ring of quotients $Q$ is a simple Artinian ring. Suppose that $e_{1}$ and $e_{2}$ are the canonical idempotents of $R$ with a 1 in one positions and zero everywhere else. Then it is easy to see that $A=e_{1} Q+R$ and $B=e_{2} Q+R$ are not subrings of $Q$. Moreover, $A+B=e_{1} Q+e_{2} Q+R=Q$ and $A \cap B=R$.

THeOREM 5.5. Let $R$ be a semi-prime right and left Goldie-ring such that $Q_{R}$ is a countably generated right $R$-module.

a) An R-module is h-divisible if and only if it is divisible in the classical sense. In particular, every $R$-module $M$ which is divisible in the classical sense is weakly cotorsion.

b) $Z(D)$ is a direct summand of $D$ whenever $D$ is divisible in the classical sense.

c) $R$ is a p.p.-ring if and only if the classes of h-divisible and divisible modules coincide.

PRoof. a) By Theorem 5.2, it remains to show that every right $R$ module, which is divisible in the classical sense, is weakly cotorsion. Since the class of modules, which are divisible in the classical sense, is closed with respect to extensions, it suffices to consider a module $D$ which is divisible in the classical sense, and to show that every epimorphism $\pi: D \rightarrow Q$ splits.

Suppose that $Q=\Sigma_{n<\omega} u_{n} R$ where $u_{0}=1$. We construct an ascending chain $\left\{V_{n}\right\}_{n<\omega}$ of submodules of $Q_{R}$ such that $V_{0}=R, V_{n}=d_{n}^{-1} R$ for some regular element $d_{n}$ of $R$ subject to the conditions $d_{0}=1, d_{n+1}=c_{n} d_{n}$ for some regular element $c_{n}$ of $R$, and $u_{n} \in V_{n}$.

Suppose that regular elements $d_{0}, \ldots, d_{n}$ and submodules $V_{0} \subseteq \ldots \subseteq V_{n}$ of $R$ have already been constructed with the desired properties. Since $Q$ is the classical right and left ring of quotients of $R$, there are $c_{n}, r_{n} \in R$ with $c_{n}$ regular such that $d_{n} u_{n+1}=c_{n}^{-1} r_{n}$. Set $d_{n+1}=c_{n} d_{n}$ and $V_{n+1}=d_{n+1}^{-1} R$. Since $d_{n}^{-1}=d_{n+1}^{-1} c_{n}$, we obtain $V_{n} \subseteq V_{n+1}$. Moreover, $u_{n+1}=d_{n}^{-1} c_{n}^{-1} r_{n}=$ $=d_{n+1}^{-1} r_{n} \in V_{n+1}$. Therefore, $Q=\cup_{n<\omega} V_{n}$.

Choose $s_{0} \in D$ with $\pi\left(s_{0}\right)=1 \in R$, and define $\delta_{0}(1)=s_{0}$. Suppose that we have already defined morphisms $\left\{\delta_{i}: V_{i} \rightarrow D \mid i=0, \ldots, n\right\}$ such that $\delta_{j} \mid V_{i}=\delta_{i}$ for all $0 \leq i<j \leq n$ and $\pi \delta_{i}=1_{V_{i}}$ for all $i=0, \ldots, n$. Let $s_{n}=\delta_{n}\left(d_{n}^{-1}\right)$. Because $D$ is divisible in the classical sense, there is $s_{n+1} \in D$ such that $s_{n+1} c_{n}=s_{n}$. Since $V_{n+1}=d_{n+1}^{-1} R$ is a free $R$-module, setting $\delta_{n+1}\left(d_{n+1}^{-1}\right)=s_{n+1}$ induces an $R$-module map $\delta_{n+1}: V_{n+1} \rightarrow D$. In view of $d_{n}^{-1}=d_{n+1}^{-1} c_{n}$, we obtain $\delta_{n+1}\left(d_{n}^{-1}\right)=\delta_{n+1}\left(d_{n+1}^{-1}\right) c_{n}=s_{n+1} c_{n}=s_{n}=\delta_{n}\left(d_{n}^{-1}\right)$; and $\delta_{n+1} \mid V_{n}=\delta_{n}$. Moreover, $\pi \delta_{n+1}\left(d_{n+1}^{-1}\right) c_{n}=\pi \delta_{n+1}\left(d_{n+1}^{-1} c_{n}\right)=\pi \delta_{n+1}\left(d_{n}^{-1}\right)=$ 
$=\pi \delta_{n}\left(d_{n}^{-1}\right)=d_{n}^{-1}=d_{n+1}^{-1} c_{n}$. Since $Q$ is non-singular, $\pi \delta_{n+1}\left(d_{n+1}^{-1}\right)=d_{n+1}^{-1} ;$ and $\pi \delta_{n+1}=1_{V_{n+1}}$. Then, $\delta: Q \rightarrow D$ defined by $\delta(x)=\delta_{n}(x)$ if $x \in V_{n}$ satisfies $\pi \delta=1_{Q}$.

b) follows from Corollary 4.6 and part a).

c) If $R$ is a p.p.-ring, then h-divisible modules are divisible by Corollary 4.2. Conversely, every divisible module $D$ is divisible in the classical sense. By a), $D$ is h-divisible.

On the other hand, if h-divisible modules and divisible modules coincide, then the class of divisible modules is closed with respect to epimorphic images. By Lemma 3.1, $R$ is a p.p.-ring.

If $R=\operatorname{Mat}_{2}(Z[x])$, then the class of h-divisible modules coincides with the class of modules which are divisible in the classical sense. However, there exist h-divisible modules which are not divisible since $R$ is not a p.p.-ring [6, Theorem 8.17].

We now give an example that there may exist weakly cotorsion modules which are divisible in the classical sense but not h-divisible if $R$ is not semiprime:

EXAMPLE 5.6. Let $R$ be any lower triangular matrix ring over a field $F$ of order at least 2. By [8, Theorem 5.27], $R$ is right and left hereditary. Hence, h-divisible modules are injective. However, since $R$ is Artinian, all regular elements of $R$ are units. Thus, every $R$-module is divisible in the classical sense. Furthermore, another application of [8, Theorem 5.27] yields that $Q^{r}$ is flat as a right and left $R$-module. Since $Q^{r}$ also is finitely generated, it is projective. Therefore, $\operatorname{Ext}_{R}^{1}\left(Q^{r}, M\right)=0$ for all $R$-modules $M$. On the other hand, since $R$ is not semi-prime, there exist an $R$-module which is not injective.

Proposition 5.7. Let $R$ and $S$ be a rings, and $\mathcal{F}: \mathcal{M}_{R} \rightarrow \mathcal{M}_{S}$ an equivalence with inverse $\mathcal{G}$.

a) If $R$ is a semi-prime right and left Goldie-ring such that $Q / R$ is a direct sum of countably generated modules, then the same holds for $S$.

b) If $R$ has finite right Goldie-dimension, then $\mathcal{F}(D)$ is h-divisible whenever $D$ is h-divisible.

Proof. a) It remains to show that $Q(S) / S$ is a direct sum of countably generated modules. Since $Q(S)$ is the injective hull of $S$, we obtain that $\mathcal{G}(Q(S)$ ) is the injective hull of $\mathcal{G}(S)$. Because $\mathcal{G}(S)$ is a finitely generated projective $R$ module, there is a projective module $P$ such that $\mathcal{G}(S) \oplus P \cong R^{n}$ for some 
$n<\omega$. Then, $\mathcal{G}(Q(S)) \oplus E(P) \cong Q^{n}$. Thus, $\mathcal{G}(Q(S)) / \mathcal{G}(S) \oplus E(P) / P \cong(Q / R)^{n}$. By Kaplansky's Theorem, $\mathcal{G}(Q(S)) / \mathcal{G}(S)=\oplus_{I} U_{i}$ where each $U_{i}$ is countably generated. Since $\mathcal{F}(R)$ is finitely generated, $\mathcal{F}\left(U_{i}\right)$ is countably generated.

b) Since $R$ has finite right Goldie-dimension, $D$ is an epimorphic image of an injective.

Combining the last two results shows that the property that the classes of divisible and h-divisible modules coincide is not Morita-invariant.

\section{REFERENCES}

[1] U. Albrecht, Two-sided submodules of $Q^{r}$, Houston Journal of Mathematics, 33 (1), pp. 103-123.

[2] U. ALBRECht, Finitely Presented Modules over Right Non-Singular Rings; Rend. Sem. Math. Padova, 120 (2008), pp. 45-58.

[3] U. ALBREChT - A. FACCHINI, Mittag-Leffler modules over non-singular rings; Rend. Sem. Mat. Univ. Padova, 95 (1996), pp. 175-188.

[4] U. ALBRECHT - J. DAUNS - L. FuChS, Torsion-freeness and non-singularity over right p.p.-rings; Journal of Algebra, 285 (2005), pp. 98-119.

[5] U. Albrecht - J. TRilifaJ, Cotilting classes of torsion-free modules J. Alg and Appl., 5 (6) (2006), pp. 747-764.

[6] A. W. Chatters - C. R. Hajarnavis, Rings with Chain Conditions; Pitman Advanced Publishing 44; Boston, London, Melbourne (1980).

[7] L. Fuchs - L. SAlce, Modules over Non-Noetherian Domains; Mathematical Surveys and Monographs 84; Amer. Math. Soc. (2000).

[8] K. GoodeARL, Ring Theory; Marcel Dekker; New York, Basel (1976).

[9] A. HATTORI, A foundation of torsion theory for modules over general rings; Nagoya Math. J., 17 (1960), pp. 147-158.

[10] E. Matlis, Divisible modules; Proc. Amer. Math. Soc., 11 (1960), pp. 385-391.

[11] J. Rotman, An Introduction to Homological Algebra; Academic Press, London (1979).

[12] B. Stenström, Rings of Quotients; Lecture Notes in Math., 217; Springer Verlag, Berlin, Heidelberg, New York (1975).

Manoscritto pervenuto in redazione il 23 aprile 2009. 